\title{
Model of suspension displacement in a porous medium
}

\author{
Liudmila Kuzmina ${ }^{1}$, and Yuri Osipov, ${ }^{2, *}$ \\ ${ }^{1}$ National Research University Higher School of Economics, 101000 Moscow, Russia \\ ${ }^{2}$ Moscow State University of Civil Engineering, Yaroslavskoe shosse, 26, Moscow, 129337, Russia
}

\begin{abstract}
The displacement of monodisperse suspension by clean water in a porous medium, accompanied by the formation of deposit is considered. A flow of water is supplied at the inlet of the porous medium filled with suspension. The suspension is displaced by water moving at a constant velocity. A mathematical model of deep bed filtration of suspension in a porous medium based on size-exclusion mechanism of particle retention and pore clogging is formulated. It is proved that in the suspension area the solution depends only on time, and in the clean water area - only on the distance to the porous medium inlet. For constant initial conditions an exact solution of the problem is constructed. In the case of linear filtration coefficient, the analytic solution is given in explicit form. The properties of the obtained solutions are analyzed.
\end{abstract}

\section{Introduction}

Filtration of suspensions and colloids in a porous medium occurs in many fields of science and technology. For the construction of waterproof walls of underground hazardous waste storage facilities, filtration of the grout solution in the porous rock is used [1]. The transfer of suspended particles and the retention of particles in pores is important in the filtration of water [2], wastewater treatment and liquid industrial wastes [3]. In the oil industry, the productivity of wells can be significantly reduced by pumping sea or formation water [4] due to the transfer and sedimentation of suspended particles in the porous soil.

The mathematical formulation of the filtration problem first appeared in the study of the Director of the Water Supply Administration in Tokyo, T. Iwasaki [5] in 1937. The article considered the process of deep bed filtration, in which filtration for a long time occurs throughout the whole porous medium, and not only in its surface layer. The basic filtration equations are derived in [6]. The equation of mass balance of suspended and retained particles, and the equation for the formation of a deposit with unknown concentrations of suspended and retained particles consider the suspension flow in a porous medium. The first equation is an analog of the continuity equation, considering the immobility of the retained particles. The second equation determines that the rate of the deposit formation is proportional to the concentration of suspended particles in the suspension. The coefficient of proportionality is called the filtration coefficient. In the simplest model, the filtration

\footnotetext{
*Corresponding author: yuri-osipov@ mail.ru
} 
coefficient is assumed to be constant; in the general case, it depends on the retained particles concentration. Retained particles block the small pores of the porous medium, and as the number of free pores decreases, the rate of deposit formation decreases.

Depending on the type of suspension and the properties of the porous medium, the process of particle retention is determined by viscosity, dispersion, electrostatic and gravitational forces. The corresponding mathematical models are suitable for different types of filtration [7, 8]. The probabilistic models of particle transport in a porous medium are developed successfully $[9,10]$. The dependence of the porous medium properties on the retained particles concentration complicates the model equations [11, 12].

The ratio of particle and pore sizes is important for the particle retention. If the particle and pore size distributions overlap, the mechanic-geometric mechanism of particle capture and deposit formation plays the main role in the retention of particles by a porous medium [13]. It is assumed that the particles freely pass through pores larger than the particle sizes and get stuck at the inlet of small pores whose diameter is smaller than the particle size. The delayed particles cannot be knocked out of the pores by the fluid flow or other particles and form a deposit.

The paper studies the displacement of a suspension from a porous medium by the water flow. In Section 2, the general mathematical model of the filtration of a monodisperse suspension in a homogeneous porous medium is reduced to an ordinary differential equation of the first order. An exact solution for constant initial conditions is constructed in Section 3. In Section 4, the analytical solution is obtained in explicit form for the linear filtration coefficient. Section 5 is devoted to the discussion of results and conclusions.

\section{Mathematical model}

The displacement of a monodisperse suspension by clean water in a porous medium is determined by two first-order partial differential equations. In the domain

$$
\Omega=\{0<x<1, t>0\}
$$

the dimensionless concentrations of suspended $C(x, t)$ and retained $S(x, t)$ particles satisfy the system of equations

$$
\frac{\partial C}{\partial t}+\frac{\partial C}{\partial x}+\frac{\partial S}{\partial t}=0
$$

$$
\frac{\partial S}{\partial t}=\Lambda(S) C
$$

with the boundary and initial conditions

$$
\begin{gathered}
x=0: C=0, \\
t=0: C=C_{0}(x), S=S_{0}(x) .
\end{gathered}
$$

Here the filtration coefficient $\Lambda(S)$ and the initial particles concentrations $C_{0}(x), S_{0}(x)$ are continuous positive functions.

Condition (3) corresponds to the injection of clean water without suspended particles into the a porous medium. Condition (4) means that at the initial time the entire porous medium is filled with suspended and retained particles. 
The general form of the solution of the problem (1) - (4) is shown in Figure 1. According to the method of characteristics (see [14, Section 6.4]), the solution has the form: in the suspension zone $\Omega_{1}=\{0<x<1,0<t<x\}-C_{1}>0, S_{1}>0$; in the water zone $\Omega_{2}=\{0<x<1, t>x\}-C_{2}=0, S_{2}>0$.

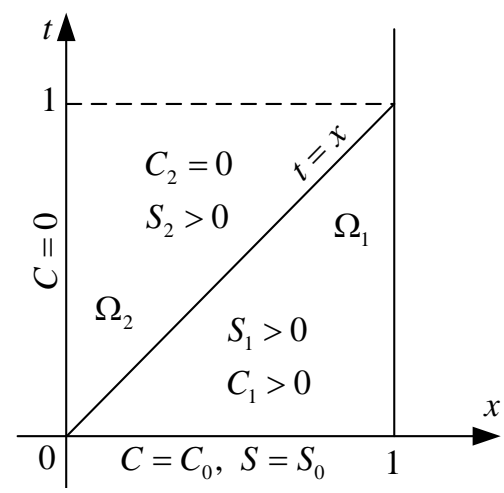

Fig. 1. The scheme of the solution for the problem (1)-(4).

The characteristic line $t=x$ is the mobile boundary between water and suspension. At the boundary, the solution $C(x, t)$ has a strong discontinuity (the suspended particles concentration undergoes a discontinuity), and the solution $S(x, t)$ has a weak discontinuity (the retained particles concentration is continuous and its derivatives are discontinuous).

Consider the system (1) - (4) in the domain $\Omega_{1}$. In the characteristic variables

$$
\tau=t-x, x=x
$$

the system (1) - (4) takes the form

$$
\begin{gathered}
\frac{\partial C}{\partial x}+\frac{\partial S}{\partial \tau}=0 ; \\
\frac{\partial S}{\partial \tau}=\Lambda(S) C ; \\
x=0: C=0 ; \\
\tau=-x: \quad C=C_{0}(x), \quad S=S_{0}(x) .
\end{gathered}
$$

Expression of $C$ from equation (7) yields

$$
C=\frac{\partial S}{\partial \tau} / \Lambda(S)
$$

Substitution of (10) into equation (6) and integration with respect to the variable $\tau$ gives

$$
S+\frac{\partial S}{\partial x} / \Lambda(S)=K(x)
$$


where $S(x, \tau), K(x)$ are unknown smooth functions.

The function $K(x)$ is obtained from the initial conditions (9)

$$
K(x)=C_{0}+S_{0}+\frac{S_{0}^{\prime}}{\Lambda\left(S_{0}\right)} .
$$

Equation (11) with the initial condition (9) has a unique solution $S(x, \tau)$ that determines the retained particles concentration $S_{1}(x, t)=S(x, t-x)$ in the domain $\Omega_{1}$. The suspended particles concentration $C_{1}(x, t)$ is obtained from relation (10).

In the characteristic variables (5) the mobile boundary between water and suspension is given by the formula $\tau=0$. The solution on the boundary

$$
S_{2}(x)=\left.S(x, \tau)\right|_{\tau=0}
$$

Formula (13) determines the retained particles concentration in the domain $\Omega_{2}$. The obtained solution is continuous in the domain $\Omega=\Omega_{1} \cup \Omega_{2}$.

\section{Exact solution for constant initial conditions}

Consider the system (1) - (4) with constant initial conditions

$$
t=0: C=1, S=q, q>0 .
$$

Since conditions (14) do not depend on the variable $x$, the solution of the system in the domain $\Omega_{1}$ also does not depend on $x$. A solution of the problem (1) - (3), (14) is obtained in the form $C=C(t), S=S(t)$. Equation (1) takes the form

$$
\frac{\partial C}{\partial t}+\frac{\partial S}{\partial t}=0
$$

Integration of the equation (15) with respect to the variable $t$ yields

$$
C+S=k=\text { const } .
$$

The constant $k$ is obtained from the initial conditions (14)

$$
k=1+q .
$$

The unknown $C$ is expressed from the equation (16)

$$
C=1+q-S .
$$

Substitution of (17) into (2) gives

$$
\frac{\partial S}{\partial t}=\Lambda(S)(1+q-S)
$$

The solution of the equation (18) with the condition (14) 


$$
\int_{q}^{S(t)} \frac{d S}{\Lambda(S)(1+q-S)}=t
$$

Formula (19) determines the retained particles concentration $S(t)$ in the domain $\Omega_{1}$. The suspended particles concentration in the domain $\Omega_{1}$ is given by the relation (17).

In the domain $\Omega_{2}$, the solution of the problem (1) - (3), (14) has the form

$$
C=0 ; S=S(x) \text {. }
$$

\section{Calculation of the problem with a linear filtration coefficient}

Consider the system (1) - (3), (14) with a linear filtration coefficient

$$
\Lambda(S)=a+b S .
$$

In this case the integral (19) is calculated in explicit form and the solution in the domain $\Omega_{1}$ has the form

$$
S(t)=\frac{(1+q)(a+b q) e^{(a+b(1+q)) t}-a}{(a+b q) e^{(a+b(1+q)) t}+b} ; C(t)=\frac{a+b(1+q)}{(a+b q) e^{(a+b(1+q)) t}+b} ; \quad(x, t) \in \Omega_{1} .
$$

The solution in the domain $\Omega_{2}$

$$
S(x)=\frac{(1+q)(a+b q) e^{(a+b(1+q)) x}-a}{(a+b q) e^{(a+b(1+q)) x}+b} ; C=0 .
$$

Numerical calculation of the problem by the method of finite differences (solid line) and calculation by exact formulas (dashed lines) are performed for $q=0.5, a=2, b=-1$.

In Figures 2 and 3 the graphs of the suspended $C$ and retained $S$ particles concentrations are shown $a$ ) at a fixed time $t=0.5$ and $b$ ) in the middle of the porous medium sample $x=0.5$.
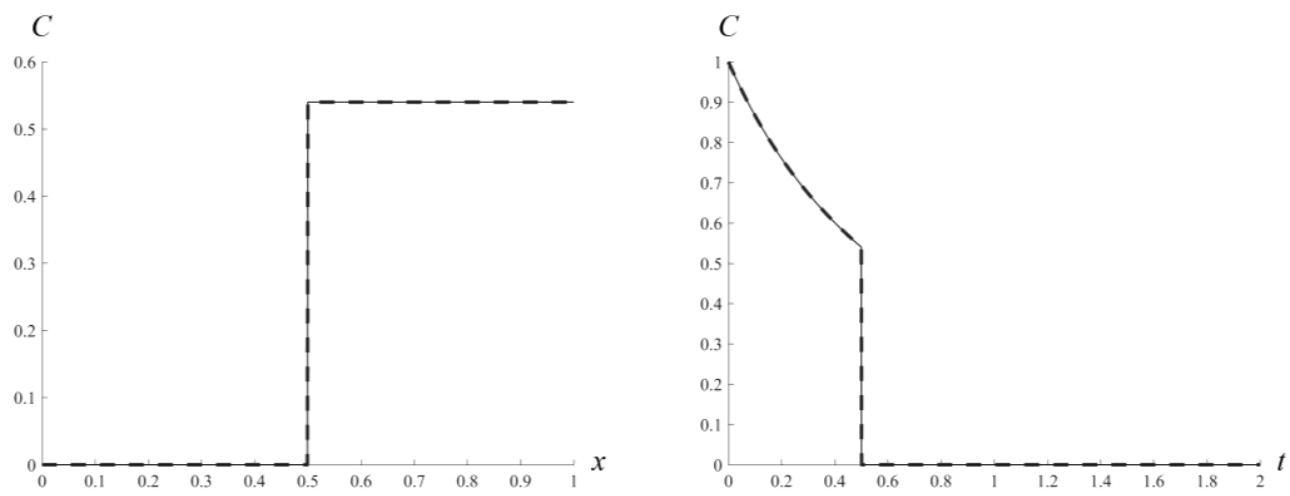

Fig. 2. The suspended particles concentration $C(x, t) a)$ for $t=0.5 ; b)$ for $x=0.5$. 

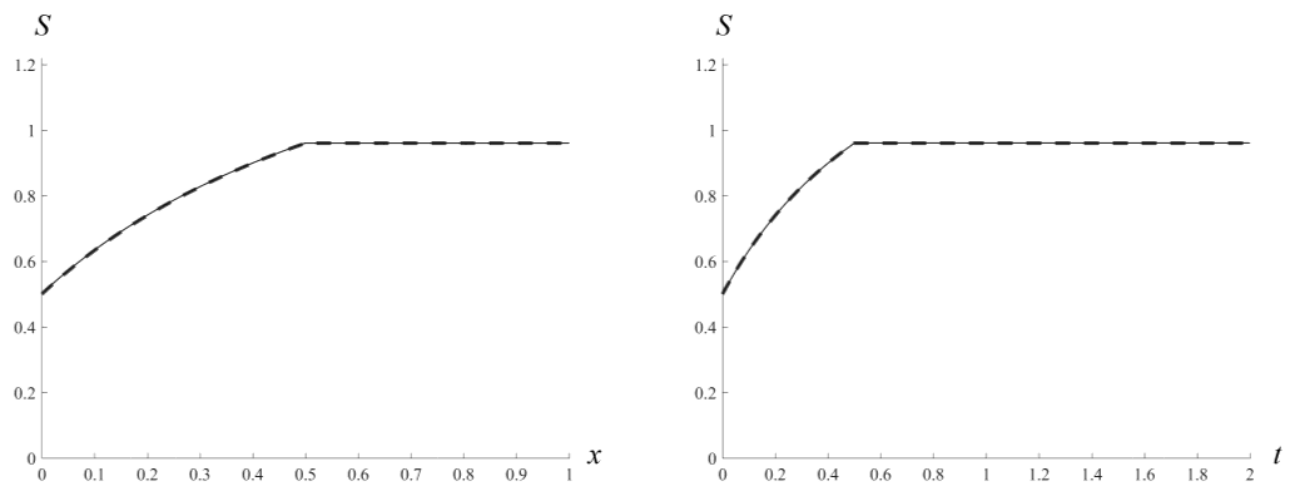

Fig. 3. The retained particles concentration $S(x, t) a)$ for $t=0.5 ; b)$ for $x=0.5$.

\section{Discussion and conclusions}

The solution of the filtration problem for the displacement of the suspension by water flow in a porous medium is obtained. In the general case, the system of partial differential equations is reduced to one ordinary differential equation of the first order. Under constant initial conditions, the solution is written in implicit form. For the linear filtration coefficient, an analytical solution is obtained in explicit form. The solution coincides with the numerical calculation (Figures 2,3).

Under constant initial conditions, the solution is a function of one variable. In the domain $\Omega_{1}$, the solution depends only on $t$, and in the domain $\Omega_{2}-$ only on $x$. In the suspension zone $\Omega_{1}$ the concentrations of suspended and retained suspension particles depend only on time; at a fixed time $t=t_{0} \leq 1$, the concentrations are constant at all points of the porous medium filled with suspension. With the arrival of clean water, the concentration of suspended particles becomes zero, and the retained particles concentration is constant over time. Since water moves from the porous medium inlet $x=0$ to the outlet $x=1$ at a constant velocity $v=1$, the duration of the filtration process is proportional to the distance from the inlet, and the retained particles concentration increases with increasing $x$. Accordingly, the suspended particles concentration decreases.

In the domain $\Omega_{1}$, consider the solution $S(t)$ for large time. If the filtration coefficient $\Lambda(S)$ is positive for $S \in[q ; 1+q]$, then formulas (19), (17) give

$$
\lim _{t \rightarrow \infty} S(t)=1+q ; \lim _{t \rightarrow \infty} C(t)=0 .
$$

For the linear filtration coefficient (21), the relations (24) follow directly from formulas (22).

In the domain $\Omega_{1}$, the time $t$ varies on the interval [0;1]. Consequently, the limiting values (24) are not attained. The maximum value of the solution $S(t)$ and the minimum value of $C(t)$ are attained at the porous medium output $x=1$

$$
\max _{t} S(t)=S(1)<1+q ; \min _{t} C(t)=C(1)>0 .
$$

Numerical calculation of the periodic pollution and cleaning the porous media by the fluid flow changing its direction was carried out in [15]. 
Note that the obtained solution can be used in the analysis and processing of laboratory and field experiments [16].

The authors are grateful to Yu.P. Galaguz for numerical modeling and fruitful discussion.

\section{References}

1. M. Tsuji, S. Kobayashi, S. Mikake, T. Sato, H. Matsui, Post-Grouting Experiences for Reducing Groundwater Inflow at $500 \mathrm{~m}$ Depth of the Mizunami Underground Research Laboratory, Japan, Procedia Engineering, 191 (2017) p.543-550.

2. C. Noubactep, S. Care, Dimensioning metallic iron beds for efficient contaminant removal, Chemical Engineering Journal, 163 (2010), p.454-460.

3. N. Tufenkji, D.R. Dixon, R. Considine, C.J. Drummond, Multi-scale Cryptosporidium /sand interactions in water treatment, Water Research, 40 (2006) p.3315-3331.

4. P. Bedrikovetsky, Mathematical theory of oil and gas recovery: with applications to ex-USSR oil and gas fields (Kluwer Academic, Dordrecht, 1993).

5. T. Iwasaki, Some notes on sand filtration, Journal American Water Works Association, 29 (1937) p.1591-1602.

6. J.P. Herzig, D.M. Leclerc, P. Legoff, Flow of suspensions through porous media application to deep filtration, Industrial and Engineering Chemistry, 62 (1970) p.8-35.

7. V. Jegatheesan, S. Vigneswaran, Deep bed filtration: mathematical models and observations, Critical Reviews in Environmental Science and Technology, 35, 6 (2005) p.515-569.

8. C.V. Chrysikopoulos, V.I. Syngouna, Effect of gravity on colloid transport through water-saturated columns packed with glass beads: Modeling and experiments, Environmental Science and Technology, 48 (2014) p 6805-6813.

9. A.C. Payatakes, R. Rajagopalan, C. Tien, Application of porous media models to the study of deep bed filtration, Canadian Journal of Chemical Engineering, 52 (1974) p.722-731.

10. A.A. Shapiro, Elliptic equation for random walks. Application to transport in microporous media, Physica A, 375 (2007) p.81-96.

11. Z. You, P. Bedrikovetsky, L. Kuzmina, Exact solution for long-term size exclusion suspension-colloidal transport in porous media, Abstract and Applied Analysis, 2013 (2013) ID 680693.

12. L.I. Kuzmina, Yu.V. Osipov, Asymptotics of the filtration problem for suspension in porous media, Vestnik MGSU, 1 (2015) p.54-62.

13. A. Santos, P. Bedrikovetsky, A stochastic model for particulate suspension flow in porous media, Transport in Porous Media, 62 (2006) p.23-53.

14. B.L. Rozhdestvenskii, N.N. Yanenko, Systems of quasilinear equations and their applications to gas dynamics (American Mathematical Society, Providence, RI, 1983).

15. Yu.P. Galaguz, G.L. Safina, Modeling of particle filtration in a porous medium with changing flow direction, Procedia Engineering, 153 (2016) p.157-161.

16. P.G. Bedrikovetsky, D. Marchesin, F. Checaira, A.L. Serra, E. Resende, Characterization of deep bed filtration system from laboratory pressure drop measurements, Journal of Petroleum Science and Engineering, 32, 3 (2001) p.167-177. 